\title{
Globalización, división internacional del trabajo y situación laboral en Centroamérica ${ }^{1}$
}

\author{
Rafael Antonio Pleitez $z^{2}$
}

\section{Resumen}

Los años noventa pueden ser caracterizados como la década de la globalización. El fin de la "Guerra Fría" significó la apertura de los países de economías socialistas al capitalismo y la esperanza del inicio de un período de paz y progreso mundial. Sin embargo, el optimismo de inicios de los noventa se fue tornando en una creciente preocupación social debido al incremento de la pobreza, el crecimiento económico con desempleo, la creciente desigualdad en la distribución del ingreso y la atomización social, expresada en conflictos étnicos y crecientes niveles de violencia ${ }^{3}$. Esta situación puede explicarse, en gran pàrte, a partir del proceso de globalización de orientación "neoliberal".

\section{Introducción}

Este artículo tiene el propósito de realizar una reflexión teórica sobre la globalización y sus consecuencias en el mundo del trabajo, tomando como ejemplo la situación laboral de Centroamé- rica. Está organizado en tres partes. En primer lugar se hace un análisis crítico sobre el proceso de globalización de orientación neoliberal. En segundo lugar se realiza una breve caracterización de los mercados laborales en Centroamérica. Y, finalmente, se hace un análisis sobre los desafíos que la

1. Ponencia presentada en la Escuela Sindical de la CCOOPV, organizado por la Fundación Paz y Solidaridad, en Valencia, del 29 al 31 de octubre de 1998.

2. Jefe del Departamento de Economía de la Universidad Centroamericana "José Simeón Cañas" (UCA) de El Salvador.

3. Estas tendencias fueron reconocidas en la Cumbre Mundial sobre Desarrollo Social organizada por la ONU, en 1995. 
globalización presenta a los trabajadores centroamericanos.

\section{El proceso de globalización con orientación "neoliberal"}

A continuación analizaremos el proceso de globalización a partir de dos ejes: uno económico y otro de carácter filosófico. El eje económico está basado en la explicación de la crisis del "fordismo" y en la comprensión de la mundialización como una etapa más del desarrollo del capitalismo. En el eje filosófico se trata de explicar el nexo social mundial y se da una visión crítica del término "neoliberalismo". Un punto por destacar es el hecho de que la globalización tiene un carácter multidimensional que no se reduce a la esfera de lo económico.

\subsection{El fordismo y su crisis}

Una forma de comprender el actual proceso de globalización, en su dimensión económica, es a partir de la crisis del "fordismo" y la instauración de un nuevo ciclo de acumulación del capital. El modelo fordista debe su nombre al empresario norteamericano Henry Ford, que tuvo la idea de producir en serie autos baratos con la introducción de cadenas de producción muy especializadas, que le posibilitaron aumentar significativamente la productividad, los salarios y las prestaciones de sus obreros. Así, el término "fordismo" fue utilizado para designar a las formaciones capitalistas que se constituyeron desde los años treinta hasta los cincuenta, alcanzando su cima en los setenta, en los países más industrializados de occidente, bajo la hegemonía de Estados Unidos (Hirsch, 1994, p. 22).

El modelo fordista se caracterizó por lo siguiente: $(a)$ la organiżación taylorista del proceso de trabajo, $(b)$ la producción en serie, y $(c)$ el consumo de masas. El taylorismo significó la destrucción de varias formas de trabajo artesanal y la introducción de técnicas eficientes de control y supervisión administrativa, optimizando los espacios y tiempos en las cadenas de producción (Hirsch, 1994, p. 23). La organización taylorista del proceso de trabajo posibilitó un extraordinario incre- mento de la productividad, incrementos en los salarios reales y la producción masiva de bienes y servicios.

El fordismo fue la base de un ciclo de acumulación del capital que descansó en la "universalización" del trabajo asalariado y en el incremento en la productividad del trabajo. La generalización del trabajo asalariado posibilitó el crecimiento de los sindicatos, que tuvieron una función central en la institucionalización de la contratación colectiva e influyeron en la formulación de políticas estatales (Hirsch, 1994, p. 25). Asimismo se incrementó la tasa de ganancia en el período fordista.

A finales de los sesenta, la organización científica del trabajo muestra sus límites para seguir aumentando significativamente la productividad del trabajo. Los sindicatos se resistieron a los intentos de una reducción importante de prestaciones y salarios. Ante crecimientos más moderados de la productividad y el mantenimiento relativo de los ingresos a la clase trabajadora, la tasa media de ganancia del capital industrial disminuyó. Ante esta situación, los capitalistas industriales buscaron alternativas. El abandono del patrón oro-dólar permitió el surgimiento de los mercados especulativos de divisas. Así se invirtieron crecientes capitales en los mercados financieros. En la actualidad, el capital financiero es el que domina a nivel mundial.

Las alternativas a la producción industrial se encontraron en la automatización de los procesos de producción y en la internacionalización de las cadenas de producción (auge de las maquilas en los países subdesarrollados debido al menor coste de la fuerza de trabajo). Por último, cabe señalar que la disminución de la rentabilidad del gran capital industrial contribuyó a sustituir el keynesianismo por un nuevo paradigma denominado "neoliberal", que respondía a las necesidades de ampliar los espacios de valorización del gran capital ${ }^{4}$.

Según Vidal Villa, la globalización económica representa la culminación a escala mundial del proceso de expansión del capitalismo, posibilitado por la tecnología moderna. Este autor destaca que

4. El keynesianismo acepta que el gobierno puede contribuir a incrementar el nivel de empleo en una economía y lograr un mayor crecimiento económico. Para ello recomienda políticas fiscales y monetarias expansivas y un rol de redistribución del ingreso por parte del gobierno. El keynesianismo fue el paradigma macroeconómico predominante hasta la década de los setenta. 
las empresas multinacionales son los agentes activos de este proceso; que el requisito básico para la existencia de una economía mundial es la libertad de movimiento de capitales, mercancías y fuerza de trabajo, proceso que es obstaculizado por la existencia de los Estados "nacionales", y es un proceso que profundiza el desarrollo desigual, aumentando la polarización entre ricos y pobres (Vidal Villa, 1996, pp. 86-104).

De acuerdo con Ander Jiménez, la globalización económica presenta una profunda tendencia a las fusiones de grandes empresas multinacionales, conformando mercados acentuadamente oligopólicos, por ejemplo Intel-Microsoft (Jiménez, 1998, pp. 148-152). Por tanto, si no se regula el proceso de fusiones en el marco de la globalización, la tendencia es a la formación de oligopolios a nivel del mercado mundial.

\subsection{La revolución tecnológica y sus consecuen- cias en el mundo del trabajo}

Los avances científicos en los campos de la microelectrónica, la biotecnología y la informática y sus aplicaciones a los procesos productivos constituyen la nueva revolución tecnológica. Esta revolución conlleva a un cambio importante en las relaciones laborales, en el sentido de fragmentar y diversificar al trabajador masivo taylorista y debilitar la organización colectiva de los trabajadores (Hirsch, 1994, p. 31). Lo que se busca es la flèxibilización e individualización del trabajo, conformando un nuevo sector privilegiado de empleados altamente calificados e involucrados en las complejas tareas de dirección y supervisión del proceso del trabajo (Hirsch, 1994, p. 31).

El período posfordista se caracteriza por prácticas de trabajo flexible; una función sociopolítica muy reducida para los sindicatos; un incremento de la subcontratación de empresas y relaciones estrechas con los proveedores, y una intervención estatal muy limitada en lo económico (Lazo, 1998, pp. 115-116). Actualmente, la ventaja competitiva más importante es el conocimiento, la capacidad de invención y la aplicación de nuevas tecnolo- gías. La mayoría de empresas nacionales en los países subdesarrollados se encuentran en clara desventaja de competencia con las empresas multinacionales, que son las líderes en investigación aplicada. Las multinacionales determinarán, en buena medida, el tipo de inserción de un país subdesarrollado en las cadenas internacionales de producción.

Frente a esta revolución tecnológica, los sindicatos tienen el desafío de pasar de una posición defensiva a una ofensiva. Los trabajadores organizados deberían anticiparse a las acciones de la empresa en términos de reconversión tecnológica y productiva, y ser críticos de las dificultades de aplicación que estos cambios traen consigo. Lo anterior requiere la capacitación de los cuadros sindicales para adecuarse a la revolución tecnológica y a los nuevos métodos de organización del proceso de trabajo. Sin embargo, la nueva revolución tecnológica conlleva dos problemas muy importantes: (a) la disminución de las necesidades de horas de trabajo vivo, y $(b)$ la creciente importancia de los trabajadores altamente calificados frente a los obreros de baja calificación.

\subsection{América Latina: el modelo de industriali- zación por sustitución de importaciones y su crisis}

Mientras que en los países más industrializados de occidente se consolidaba el modelo fordista, en América Latina se impulsó la industrialización por sustitución de importaciones (ISI). Esta estrategia fue impulsada principalmente por la CEPAL y su secretario general, Raúl Prebisch, en la década de los cincuenta. Según Prebisch, el subdesarrollo de la región latinoamericana se explicaba porque la región se había especializado en la exportación de productos primarios -agropecuarios y mineros- y la importación de productos industriales. Por lo tanto, el proceso de desarrollo de la región pasaba por la industrialización. Para lograrlo se propusó un proceso gradual de sustitución de las importaciones desde bienes de consu- 
mo hasta bienes de capital. Esta estrategia requería de una protección temporal a la industria nacional naciente y el decidido apoyo en infraestructura por parte del gobierno.

El modelo de industrialización por sustitución de importaciones (ISI) enfrentó dos grandes cuellos de botella en América Latina. Primero, la industria nacional tenía como principal mercado el interno, que estaba protegido de la competencia externa pero que económicamente era muy pequeño. Los esfuerzos de redistribución del ingreso para ampliar el mercado intemo encontraron fuertes resistencias por parte de los terratenientes y sectores oligárquicos. Segundo, se enfrentó un creciente déficit comercial debido al desestímulo a las exportaciones primarias y a las crecientes necesidades de importaciones de materias primas y maquinarias.

Una de las principales distorsiones provocadas por el modelo ISI, debido al excesivo proteccionismo, fue el surgimiento de una industria nacional con tecnología poco dinámica y de baja productividad. Además, las industrias tendieron a ser intensivas en el uso de capital y no crearon los empleos suficientes para absorber a los emigrantes rurales que llegaron a las ciudades. Esto último explica, en alguna medida, el rápido crecimiento del sector informal. También la intervención directa e indirecta del gobierno provocó un sesgo antiagrícola, que desestimuló la inversión en tan importante sector (Lazo, 1998, p. 137).

Hoy en día, América Latina tiene el desafío de cómo insertarse en las cadenas internacionales de producción con su rezago tecnológico. La vía de orientación "neoliberal" apunta a una apertura rápida y a crear los mayores incentivos para la inversión extranjera. La CEPAL propone una apertura gradual con una integración selectiva basada en una competitividad genuina, producto de un progreso tecnológico.

\subsection{Visión crítica de la llamada doctrina neoli- beral}

En 1989, Luis de Sebastián escribió un artículo donde sostenía la tesis de que el fenómeno social e ideológico denominado neoliberalismo no está en concordancia con el liberalismo clásico de los siglos XVIII y XIX, y que es más cercano a la doctrina del darwinismo social (Sebastián, 1989). A continuación retomo esta tesis para analizarla.
En el pensamiento liberal clásico, la competencia es un elemento esencial del ordenamiento económico. El potencial conflicto entre el individuoegoísta y el bien común se resuelve por la acción de la competencia. Sin competencia tendríamos una sociedad donde dominaría la ley del más fuerte (Sebastián, 1989, p. 423). Según los economistas clásicos, la competencia tendría que estar inscrita en un ordenamiento jurídico, que delimite los derechos de cada uno, y con una visión ética, que tenga en cuenta las consecuencias de mis acciones sobre los demás, en búsqueda del bien particular (Sebastián, 1989, p. 425).

Un punto por resaltar de los economistas liberales clásicos es su profunda preocupación social por la situación de la clase trabajadora dentro del proceso de la revolución industrial. Sirva, para ilustrar dicha preocupación social, la siguiente cita de Smith:

KLos sirvientes, obreros y trabajadores de diversas clases sociales componen con mucho la mayoría de toda sociedad política desarrollada. Pero lo que mejora las condiciones de la mayoría nunca puede considerarse como un incoveniente para el conjunto. Ninguna sociedad puede ser floreciente y feliz, si la mayoría de sus miembros son pobres y miserables (A. Smith, La Riqueza de las naciones, Libro I, Cap. 8. En la edición de Edwin Cannan, p. 88; tomado de Sebastián, 1989, p. 426).

A finales del siglo XIX se difunden las ideas de Herbert Spencer, considerado como el padre del "darwinismo social", que defiende el privilegio de los más fuertes como un requisito para el bien de toda la sociedad (Sebastián, 1989, p. 429). Sin embargo, la gran acumulación de riqueza y poder en pocas manos comenzó a escandalizar a la opinión pública de Estados Unidos. Así que la filosofía del darwinismo social fue sustituida por los modelos de competencia perfecta desarrollados por los neoclásicos y fueron utilizados para justificar la libertad de mercado y la no-intervención del gobierno en el área económica (Sebastián, 1989, pp. 430-31).

Desde la década de los treinta hasta los setenta se vivió un período de creciente intervención del sector público en la economía. En la década de los setenta ocurre una estanflación económica en los países capitalistas más industrializados. Esta situación económica pone en crisis al keynesianismo. 
Los déficit fiscales aumentaban y comenzaron a ser financiados captando ahorros del público. Así el gobierno se presentó como competidor del sector privado en el mercado de 'capitales. Lo anterior conduce a un encarecimiento del dinero, es decir a aumentos de las tasas de interés $y$, por ende, a una reducción de la inversión. El gobiemo deja de ser parte de la solución y se convierte en el problema, desde la perspectiva neoliberal que resurge en esa década (Sebastián, 1989, p. 432).

Esta corriente de pensamiento denominada neoliberal retoma diferentes argumentos y teorías para demostrar que la intervención del gobierno es innecesaria y contraproducente. Con base en la teoría de las expectativas racionales se rechaza el manejo expansionista de las políticas monetarias y fiscales. A la economía de la demanda keynesiana contraponen la economía de la oferta. A partir de la economía de la oferta se promueve la reducción de las regulaciones a los mercados y la reducción de impuestos directos, con el fin de estimular la producción y las ganancias de los grandes inversionistas privados. Se promueve la privatización de las empresas públicas justificada por la necesidad de reducir o eliminar los déficit fiscales, que son considerados los causantes de las presiones inflacionarias. Se ataca a los sindicatos debido a que las medidas por las cuales luchan, incremento de salarios mínimos, contratos permanentes, protección contra los despidos, son consideradas distorsiones al mercado de trabajo. Esta concepción llamada neoliberal encontró importantes nichos históricos en los gobiernos de Ronald Reagan, en Estados Unidos, y Margaret Thatcher, en el Reino Unido (Sebastián, 1989, pp. 433-34.).

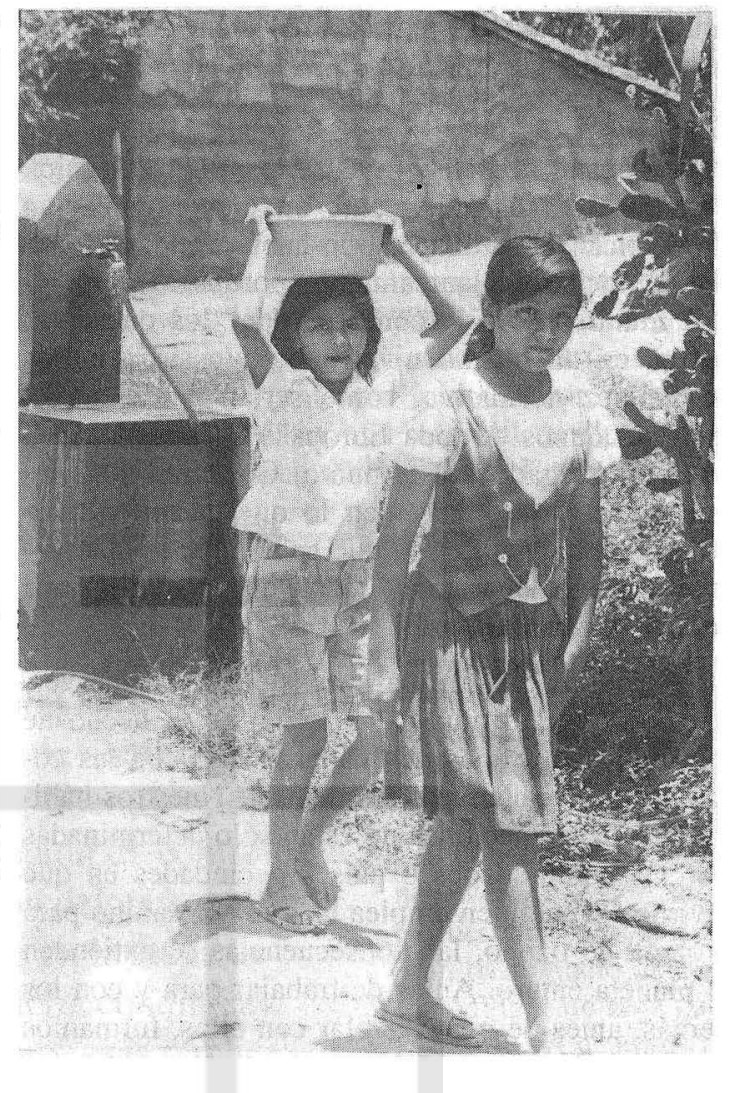

pensamiento liberal del siglo XIX (Sebastián, 1998).

\subsection{La existencia de una sociedad mundial}

¿Cómo explicaría la filosofía la existencia de una sociedad mundial? Antonio González analiza

Según Luis de Sebastián, esas son las estrategias parciales a las que falsamente se les llama neoliberalismo, pero en realidad es una ideología que favorece al capitalismo de los oligopolios. Es un darwinismo social que favorece a los que tienen más, que ha aumentado las desigualdades económicas y el número de pobres en el mundo (Sebastián, 1989, p 434). De ahí que él le denomina la revolución antiliberal, ya que deja de lado los ideales de igualdad y libertad que promovía el

Esta corriente de pensamiento denominada neoliberal retoma diferentes argumentos y teorías para demostrar que la intervención del gobierno es innecesaria y contraproducente. dos importantes posturas al respecto, como son el marxismo clásico y la acción comunicativa de Jurgen Habermas (González, 1994). Ambas le parecen insuficientes. $\mathrm{Pa}$ ra el marxismo clásico, el vínculo social, el que pone en relación a los individuos concretos, es laboralý:"Si la división del trabajo es mundial, la sociedad por antonomasia sería también una sociedad global" (González, 1994, p. 638). Sin embargo, a González esta tesis no le satisface porque deja fuera de la "sociedad 
mundial" a aquellas regiones del planeta cuyo trabajo no interesa a las redes productivas y financieras globales.

La postura de Habermas se centra en la acción comunicativa especialmente en la comunicación linguística. En definitiva, no habrá sociedad mundial desde este planteamiento porque la comunidad mundial sólo la conformarían "los que comparten en un mismo nivel evolutivo sus criterios de validez normativa, como serían por ejemplo, los ciudadanos de toda Europa" (González, 1994, p. 639). Llegado a este punto, González nos dirá que no hay que buscar en lo que el sujeto hace conscientemente o en lo que habla conscientemente el origen del vínculo social que posibilita una sociedad mundial.

Xabier Zubiri afirma la existencia de una verdadera sociedad mundial fundada en el hecho de que nuestras formas de "habérnoslas" con las cosas están afectadas por los demás. Nuestros hábitos y nuestras acciones no están sólo determinadas por las comunidades o países o ciudades en que vivimos. Si alguien emplea un aerosol dañino para la capa de ozono, las consecuencias se extienden al planeta entero. Antes de trabajar para y con los demás, antes de poder hablar con ellos, formamos una sociedad única porque nos "afectamos" los unos a los otros, aunque no estemos conscientes de ello. Con esto, González sitúa el nexo social mundial no en el nivel de la conciencia humana, sino de la sensibilidad o sentir humano.

Ahora bien, la existencia de esta sociedad mundial pone en entredicho la organización sociopolítica del Estado Moderno. La Comunidad Europea es ya una muestra de la tendencia a la superación del Estado Nación como forma de organización cuestionada en estos tiempos. Los estados naciones están en crisis. Ya no son capaces de cumplir la finalidad básica que animó su creación: el mantener a salvo las vidas de sus pobladores. Las fronteras tienden a romperse y ceden ante los embates del capital financiero, de las empresas multinacionales, de las redes de producción mundiales. El capitalismo, que hace algunos siglos originó los estados nacionales, está cada día quitándoles atribuciones, dejándolos sin poder. Nos en- contramos en un momento de transformación del orden mundial, pero la ruta aún no es clara.

\section{Breve caracterización de los mercados labo- rales en Centroamérica.}

El proceso de globalización neoliberal ha tenido una expresión concreta en la implementación de los Programas de Ajuste Estructural, impulsados por el Banco Mundial desde los ochenta. Estos programas contienen un enfoque monetarista para enfrentar los desequilibrios macroeconómicos y una concepción neoliberal de la organización económico-social, desde la cual no hay duda de la conveniencia de descentralizar las decisiones económicas en los individuos, en el contexto de un mercado que funcione sin mayor intervención del gobiemo (Pleitez, 1991, p. 66).

Las principales medidas que se implementan bajo los Programas de Ajuste Estructural son: apertura comercial (reducción significativa de los aranceles), liberalización de precios (eliminación de controles de precios), reducción del gasto público (específicamente en la etapa de estabilización macroeconómica), privatización de empresas públicas y promoción de exportaciones.

En Centroamérica, las medidas de ajuste estructural se implementaron aproximadamente desde la mitad de la década de los ochenta. Dado el contexto político, importantes conflictos bélicos en Guatemala, El Salvador y Nicaragua, estos programas no se implementaron en forma de "schock", lo que podría haber provocado enormes costos sociales y un mayor deterioro de la inestabilidad de los gobiernos en la región.

En 1998, la Facultad Latinoamericana de Ciencias Sociales (FLACSO), con sede en Costa Rica, y el Social Science Research Council, de Nueva York, publicaron un libro titulado Mercado laboral y pobreza en Centroamérica: ganadores y perdedores del ajuste estructural, los editores del libro son Edward Funkhouser y Juan Pablo Pérez Sáinz. Estos autores abordan los principales cambios en los mercados laborales durante la primera fase de los programas de ajuste estructural ${ }^{5}$. Con base en los resultados obtenidos por dicho estudio, abordaremos la evolución del empleo por sectores

5. Con base en la disponibilidad de estadísticas, los períodos analizados en cada país son los siguientes: Guatemala, 1986-1989; El Salvador, 1989-1992; Honduras, 1989-1992; Nicaragua, 1985-1993, y Costa Rica, 1989-1992. Actualizar esta investigación hasta los años más cercanos a 1998 sería una importante tarea por realizar. 
laborales; las principales características demográficas de la fuerza laboral, y la evolución de los ingresos reales promedio por sectores laborales.

\subsection{La demanda del mercado de trabajo: evo- lución del empleo por sectores laborales}

Con base en los datos del Cuadro 1 se pueden identificar las siguientes características y tendencias en el empleo por sectores laborales ${ }^{6}$.

(1) La mayor parte de la Población Económicamente Activa (PEA) centroamericana se encuentra empleada en los sectores de subsistencia agrícola e informal, que históricamente han jugado un rol de refugio de la fuerza laboral, excluida del empleo formal. Por ejemplo, los porcentajes de la Población Económicamente Activa en los sectores de subsistencia agrícola e informal son: en Guatemala, el 64 por ciento en 1986; en Honduras, el 62 por ciento en 1989; y en Nicaragua, el 54 por ciento en 1985. Estos datos reflejan la incapacidad del sector formal en generar suficiente empleo para la PEA $y$ es un factor que dificulta la organización de los trabajadores. El empleo informal muestra un crecimiento en todos los países, especialmente en El Salvador cuya tasa de crecimiento fue del 12.3 por ciento, en el período 1988-1991.

(2) Los sectores moderno agrícola y transables nuevos se encuentran orientados hacia la exportación y, por tanto, relacionados en forma directa con la dinámica de la globalización. Funkhouser y Pérez Sáinz han encontrado que "el sector moderno agrícola se muestra dinámico en todos los países, con la excepción de Nicaragua" (p. 288). El sector de transables nuevos muestra un mayor dinamismo en Honduras y Costa Rica, en el período estudiado, impulsado especialmente por las zonas francas y las maquilas. En el caso de Costa Rica, el número de trabajadores en esta actividad pasó de 5600 , en 1982 , a 46100 , en 1990 , lo que representa un incremento en el peso dentro del total del empleo industrial del 5 al 25 por ciento, respectivamente (Nowalski, et. al., 1994, p. 17) ${ }^{7}$. En Honduras se estimaron 48477 puestos de trabajo en esta actividad para 1994, lo que representa aproximadamente el 17 por ciento del total del empleo industrial (Pérez y Varela, 1995, p. 17) ${ }^{6}$. En El Salvador se calcularon en 33938 los ocupados en empresas de maquila textil, en febrero de 1996, lo que representa aproximadamente 9.3 por ciento de los ocupados en la industria (FUNDE, 1996, pp. 17-18). Cabe resaltar que la maquila predominante en la región es la textil, que demanda en gran parte mano de obra femenina y de baja calificación. La maquila textil somete a sus trabajadoras a jornadas de trabajo muy intensas, y han sido frecuentes las denuncias de maltratos e irrespeto a los derechos básicos de las trabajadoras. Es díficil creer que este sector pueda convertise en el polo de desarrollo económico-social de nuestros países.

(3) Los sectores formal y público tuvieron una función protagónica en el modelo de industrialización por sustitución de importaciones, previo a la crisis de los ochenta. Funkhouser y Pérez Sáinz destacan que el sector formal es muy dinámico en todos los países, con excepción de Nicaragua. Un dato importante es que la mayoría de la ocupación generada en este sector se concentra en las activi-

6. La definición de cada uno de los sectores laborales (moderno agrícola, subsistencia agrícola, formal, informal, público, domésticos, transables nuevos) se encuentra en una nota del autor que está al final del texto al pie de página.

7. Tomado de Funkhouser y Pérez Sáinz, 1998, p. 289.

8. Op. cit., p. 290. 
dades terciarias (comercio y servicios), lo que puede estar vinculado con el proceso de apertura comercial (Funkhouser y Pérez Sáinz, 1998, p. 290).

En relación con el empleo del sector público se identifican tres tendencias: $(a)$ crecimiento moderado en Guatemala y Honduras; (b) estancamiento en el caso de El Salvador y Costa Rica, y (c) reducción importante en el caso de Nicaragua (Funk- houser y Pérez Sáinz, 1998, pp. 290-291).

(4) En relación con el desempleo abierto se identifican tres tendencias (Funkhouser y Pérez Sáinz, 1998, pp. 292-293): (a) reducción en el caso de Guatemala y Honduras; (b) estancamiento en El Salvador, que puede ser explicado por la capacidad de absorción del sector informal, y (c) incremento moderado en Costa Rica y muy importante en Nicaragua.

\section{Cuadro 1}

Centroamérica: evolución del empleo por país y según sector laboral (porcentajes y tasas anuales de crecimiento)

\begin{tabular}{|c|c|c|c|c|c|c|c|c|c|c|}
\hline \multirow[t]{2}{*}{ Sectores laborales } & \multicolumn{2}{|c|}{ Guatemala } & \multicolumn{2}{|c|}{ El Salvador * } & \multicolumn{2}{|c|}{ Honduras } & \multicolumn{2}{|c|}{ Nicaragua } & \multicolumn{2}{|c|}{ Costa Rica } \\
\hline & 1986 & $86-89$ & 1988 & $88-91$ & 1989 & $89-92$ & 1985 & $85-93$ & 1989. & $89-92$ \\
\hline Moderno Agrícola & 12.1 & 5.0 & 4.0 & 21.4 & 6.7 & 13.2 & 5.5 & -2.0 & 9.8 & 2.5 \\
\hline Subsistencia Agrícola & 35.0 & 0.3 & 3.3 & 19.7 & 35.2 & -3.0 & 22.3 & 0.1 & 16.0 & -2.4 \\
\hline Formal & 9.6 & 18.5 & 21.0 & 5.6 & 11.5 & 11.7 & 7.7 & -2.3 & 19.0 & 6.9 \\
\hline Informal & 29.3 & 3.7 & 39.3 & 12.3 & 26.8 & 8.6 & 31.7 & 1.9 & 27.3 & 1.2 \\
\hline Público & 6.4 & 7.6 & 16.3 & -0.5 & 9.3 & 7.3 & 31.0 & -7.7 & 16.2 & 0.7 \\
\hline Domésticos & ... & --- & 6.5 & -1.5 & 5.2 & -7.1 & -.. & -- & --- & --- \\
\hline Transables nuevos & 3.9 & 1.8 & ... & ... & 1.1 & 42.0 & --- & --- & 6.8 & 6.4 \\
\hline Inclasificables & 0.3 & -2.3 & 0.3 & -6.7 & 1.1 & 30.0 & 1.0 & 34.7 & 1.2 & -18.7 \\
\hline Desempleados & 3.5 & -13.1 & 9.4 & -0.7 & 3.0 & -4.6 & 0.7 & 181.3 & 3.8 & 4.6 \\
\hline PEA Total & $\begin{array}{c}100.0 \\
(2739632)\end{array}$ & 3.7 & $\begin{array}{c}100.0 \\
(790568\end{array}$ & 7.3 & $\begin{array}{c}100.0 \\
(1520.510)\end{array}$ & 4.5 & 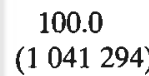 & -0.4 & $\begin{array}{c}100.0 \\
(1025.548)\end{array}$ & 2.0 \\
\hline
\end{tabular}

* Sólo áreas urbanas.

Fuente: Encuestas hogares.

Tomado de E. Funkhouser y J. Pérez Sáinz, "Ajuste Estructural, mercado laboral y pobreza en Centroamérica: una perspectiva regional”, en Mercado laboral y pobreza en Centroamérica: ganadores y perdedores del ajuste estructural, San José: FLACSO-SSRC, 1998, p. 287.

\subsection{La oferta de trabajo: características socio- demográficas}

A partir de los datos del Cuadro 2 se pueden identificar las siguientes características y tendencias de la fuerza laboral en los cinco países centroamericanos que se han estudiado (Funkhouser y Pérez Sáinz, 1998, pp. 296-297).

(1) Respecto al sexo, la región se caracteriza por un predominio de la mano de obra masculina, por ejemplo en Guatemala, el 75 por ciento de la Pobla- ción Económicamente Activa, en 1986, la conformaron los hombres. Sin embargo, la tendencia es hacia una creciente feminización de la fuerza laboral, proceso más acentuado en el caso salvadoreño ${ }^{9}$.

(2) En relación con la edad, la región se caracteriza por un predominio de los trabajadores mayores de 25 años, pero se evidencia una tendencia a una creciente incorporación de "jóvenes" en los casos de Honduras y El Salvador. Se puede objetar que el estudio de Funkhouser y Pérez Sáinz seleccionó una

9. Lo que puede explicarse debido a la cobertura sólo urbana de las encuestas de hogares utilizadas y al fenómeno de la gran emigración al exterior, sobre todo de hombres, en los últimos años. 
edad muy baja - 25 años - para analizar la dimensión etárea de la fuerza laboral en la región.

(3) Lo que destaca en relación con la escolaridad de la Población Económicamente Activa es su bajo nivel. En Guatemala, en 1986, el 83 por ciento de la PEA había cursado la primaria o menos, y en Nicaragua, en 1985, el 75 por ciento de la PEA sólo habían estudiado la primaria o menos. Entre 1985 y 1992 se identifica una tendencia a una mayor escolaridad en todos los países analizados, pero el desafío es enorme dado los niveles tan ba- jos de escolaridad, con la excepción de Costa Rica.

(4) En cuanto a la incorporación de los “jefes" y "no jefes" de hogar a los mercados de trabajo, la tendencia predominante en la región es hacia una mayor participación de los no-jefes de hogar, con la excepción de Nicaragua y Costa Rica. En otras palabras, los hogares en Centroamérica están consolidando la tendencia a la incorporación de un segundo miembro del hogar al mercado de trabajo, para complementar el ingreso principal.

\section{Cuadro 2}

Centroamérica: estructura y evolución de la fuerza laboral por país y según características sociodemográficas (porcentajes y tasas anuales de crecimiento)

\begin{tabular}{|c|c|c|c|c|c|c|c|c|c|c|}
\hline \multirow{2}{*}{$\begin{array}{c}\text { Características } \\
\text { Socio-demográficas }\end{array}$} & \multicolumn{2}{|c|}{ Guatemala } & \multicolumn{2}{|c|}{ El Salvador* } & \multicolumn{2}{|c|}{ Honduras } & \multicolumn{2}{|c|}{ Nicaragua } & \multicolumn{2}{|c|}{ Costa Rica } \\
\hline & 1986 & $86-89$ & 1988 & $89-92$ & 1989 & $89-92$ & 1985 & $85-93$ & 1989 & $89-92$ \\
\hline \multicolumn{11}{|l|}{ Sexo } \\
\hline Hombres & 75.5 & 2.0 & 58.7 & 4.7 & 70.4 & 3.6 & 67.0 & -0.5 & 75.4 & -0.3 \\
\hline Mujeres & 24.5 & 9.1 & 41.3 & 11.0 & 29.6 & 6.5 & 33.0 & -0.2 & 24.6 & 3.8 \\
\hline \multicolumn{11}{|l|}{ Edad } \\
\hline 24 años y menos & 36.7 & 3.6 & 26.2 & 8.3 & 33.5 & 4.8 & 32.5 & -0.7 & 30.9 & -1.3 \\
\hline 25 años y más & 63.3 & 3.7 & 73.8 & 6.9 & 66.5 & 4.3 & 67.5 & -0.2 & 69.1 & 3.5 \\
\hline \multicolumn{11}{|l|}{ Escolaridad } \\
\hline Primaria y menos & 83.3 & 2.8 & 56.2 & 5.9 & 60.0 & -2.0 & 75.6 & -3.4 & 58.3 & -4.4 \\
\hline Más de primaria & 16.7 & 9.0 & 43.8 & 9.8 & 40.0 & 14.2 & 24.4 & 9.7 & 41.7 & 3.9 \\
\hline \multicolumn{11}{|l|}{ Jefatura } \\
\hline Jefes & 48.8 & 2.0 & 45.4 & 4.4 & 47.7 & 2.5 & 42.1 & 0.3 & 47.8 & 2.1 \\
\hline No jefes & 51.2 & $5.0^{\circ}$ & 56.6 & 10.0 & 52.3 & 16.3 & 57.9 & -0.9 & 52.2 & 1.9 \\
\hline PEA Total & $\begin{array}{r}100.0 \\
(2739\end{array}$ & 3.7 & $\begin{array}{c}100.0 \\
(790568)\end{array}$ & 7.3 & $\begin{array}{c}100.0 \\
(1520510)\end{array}$ & )$^{4.5}$ & $\begin{array}{c}100.0 \\
(1041294)\end{array}$ & )$^{-0.4}$ & $\begin{array}{c}100.0 \\
(1025548)\end{array}$ & 2.0 \\
\hline
\end{tabular}

* Sólo áreas urbanas.

Fuente: Encuesta de hogares.

Tomado de E. Funkhouser y J. Pérez Sáinz, "Ajuste estructural, mercado laboral y pobreza en Centroamérica: una perspectiva regional", en Mercado laboral y pobreza en Centroamérica: ganadores y perdedores del ajuste estructural, San José: FLACSO-SSRC, 1998, pp. 294-295.

\subsection{Evolución de los ingresos reales promedio, según sectores laborales}

Con base en los datos del Cuadro 3 se pueden identificar las siguientes características y tendencias de los ingresos promedios reales, según los sectores laborales, durante los períodos estudiados (Funkhouser y Pérez Sáinz, 1998, p. 297-308).

(1) En general, los datos muestran un deterioro en los ingresos reales promedio en los cinco países durante los períodos estudiados. Sin embargo, en 
los casos de Guatemala y Costa Rica, el deterioro de los ingresos reales promedio ha sido mínimo; mientras que en El Salvador y Honduras el deterioro ha sido más claro, especialmente en el caso salvadoreño.

(2) A nivel de sectores laborales, el modemo agrícola resulta un sector perdedor ${ }^{10}$ en los períodos estudiados con la excepción de Costa Rica. El sector transable nuevo aparece como ganador sólo en el caso de Honduras. El sector informal aparece como perdedor a excepción de Guatemala y Costa Rica, siendo el caso salvadoreño el que presenta el mayor deterioro. Cabe señalar que en el sector informal de Costa Rica, las actividades dinámicas y no meramente de subsistencia tienen un mayor peso que en los otros países de la región. El sector de subsistencia agrícola es perdedor, con la excepción de Honduras, probablemente por la importancia del café en la situación del campesinado hondureño. El sector público es un claro perdedor en todos los países.

En suma, la tendencia predominante es que la mayoría de la fuerza laboral ha sufrido un deterioro en sus ingresos reales promedio, en el periodo del ajuste estructural que se ha estudiado.

\section{Cuadro 3}

Centroamérica: evolución del ingreso mensual real promedio por país y según sectores laborales (tasas anuales de crecimiento)

\begin{tabular}{lccccc}
\hline Sectores laborales & $\begin{array}{c}\text { Guatemala } \\
(1986-89)\end{array}$ & $\begin{array}{c}\text { El Salvador* } \\
(1988-91)\end{array}$ & $\begin{array}{c}\text { Honduras } \\
(1989-92)\end{array}$ & $\begin{array}{c}\text { Nicaragua } \\
(1985-93)\end{array}$ & $\begin{array}{r}\text { Costa Rica } \\
(1989-92)\end{array}$ \\
\hline Moderno Agrícola & -10.6 & -8.2 & -4.0 & -2.2 & 1.8 \\
Subsistencia Agrícola & -1.0 & -7.3 & 2.2 & -1.4 & -2.0 \\
Formal & 1.4 & -7.5 & -3.7 & 7.6 & -0.6 \\
Informal & 2.6 & -8.7 & -3.5 & -0.7 & 0.2 \\
Público & -1.0 & -6.9 & -8.2 & -2.5 & -0.5 \\
Doméstico & - & -2.9 & -1.9 & - & -2.8 \\
Transables nuevos & -0.5 & - & 3.1 & -3.2 & -4.6 \\
Inclasificables & 33.4 & 27.1 & -19.4 & & -0.3 \\
\hline PEA ocupada total & -0.7 & -8.1 & -3.6 & -2.1 & \\
\hline
\end{tabular}

* Sólo áreas urbanas.

Fuente: Encuesta de hogares.

Tomado de E. Funkhouser y J. Pérez Sáinz, "Ajuste estructural, mercado laboral y pobreza en Centroamérica: una perspectiva regional", en Mercado laboral y pobreza en Centroamérica: ganadores y perdedores del ajuste estructural. San José: FLACSO-SSRC, 1998, p. 298.

3.4. Cambios más importantes en el mercado laboral durante los primeros años del ajuste estructural

A partir de los resultados de la investigación coordinada por Funkhouser y Pérez Sáinz (1998, p. 331) se pueden identificar los siguientes cam- bios: (1) Los sectores orientados a la exportación, especialmente los transables nuevos, se muestran dinámicos en la generación de empleo. (2) La fuerza laboral de la región muestra una creciente feminización, y un mayor nivel de escolaridad. (3) Un mayor número de miembros de los hogares en

10. Funkhouser y Pérez Sáinz (1998) definen a un sector como ganador con el ajuste si el ingreso real promedio ha sido superior al promedio global, y perdedor si su promedio ha sido inferior (p. 297). 
Centroamérica se están incorporando al mercado laboral, para complementar el ingreso del "jefe de familia". (4) Los primeros años de implementación de los programas de ajuste estructural han generado más sectores perdedores —en términos del deterioro de los ingresos reales promedio- que ganadores. El sector público es el sector que muestra claramente un deterioro de sus ingresos reales promedio, en los cinco países estudiados. En definitiva, la mayoría de la fuerza laboral ha sufrido un deterioro en sus ingresos reales.

El proceso de globalización y su expresión concreta en los programas de ajuste estructural han conducido a hacer reformas a la legislación laboral, orientadas a flexiblizar las relaciones laborales, como por ejemplo los procedimientos de despidos, indemnizaciones, contratos por plazo determinado y fijación de salarios. La reforma del Código de Trabajo panameño, en 1995, es una muestra clara de flexibilización de las relaciones laborales (Pérez Sáinz y Allen Cordero, 1997, pp. 57-58). Asimismo, en la legislación laboral se observan también reformas referidas a los derechos de organización de los trabajadores, tal es el caso de la reforma al Código de Trabajo de El Salvador, en 1994. Estas reformas, orientadas a consagrar la libertad sindical, han sido una respuesta política a la solicitud que la AFL-CIO presentó ante el Congreso de Estados Unidos para exluir del Sistema General de Preferencias a los países que violaran las libertades sindicales (Pérez Sáinz y Allen Cordero, 1997, p. 61)"

4. Reflexiones sobre los retos que la globalización presenta a los trabajadores centroamericanos

En este artículo hemos analizado la globalización en su dimensión económica, a partir de la crisis del modelo "fordista". Bajo el modelo fordista, que predominó en los países más industrializados de occidente hasta los años setenta, ocurrió un incremento importante de la productividad del trabajo, crecieron los salarios y la tasa de ganancia media en el sector industrial, y se fortalecieron los sindicatos.

Ante la crisis del fordismo, en los años setenta, se desarrollaron tres alternativas: $(a)$ creciente inversión en los mercados financieros, (b) automatización de los procesos de producción industriales, y $(c)$ internacionalización de las cadenas de producción - auge de las maquilas en los países subdesarrollados-. La superación del "fordismo" fue posibilitado por una revolución tecnológica que provocó importantes cambios en las relaciones laborales, tales como: (a) la diversificación del trabajador homogéneo y masivo taylorista, y (b) el

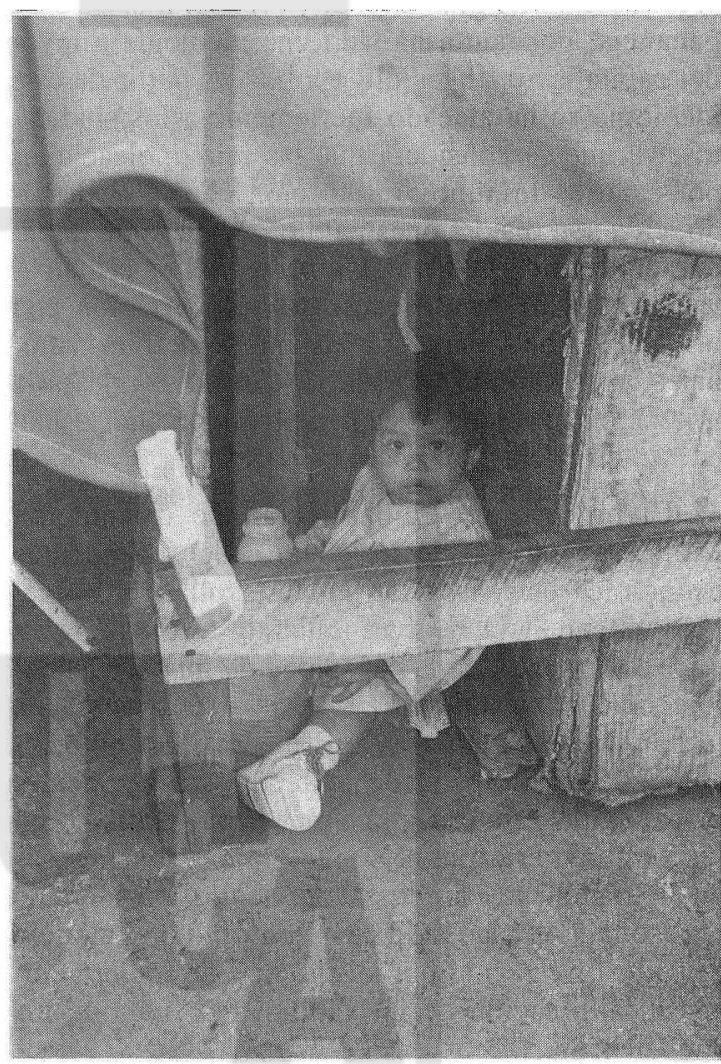

11. El desarrollo de las maquilas en la región ha sido, en buena medida, amparado a este Sistema Gcneral de Preferencias, que le ofrece un trato preferencial a las importaciones que Estados Unidos realiza de los países de Centroamérica y el Caribe. Cabe destacar que el capítulo V del "Trade and Tariff Act of 1984" establece cinco garantías laborales que deben ser respetadas para acceder a las ventajas de este sistema. Las garantías son: derecho a asociación de los trabajadores, decrecho a sindicalización, prohibición de trabajos forzosos, prohibición de utilización de menores y un régimen aceptable de condiciones de trabajo. (Tomado de Pérez Sáinz y Allen Cordero, 1997, p. 61.) 
debilitamiento de la organización colectiva del trabajo. Así, el período posfordista se caracterizó por prácticas de flexibilización e individuación del trabajo; una reducción de la función sociopolítica de los sindicatos; incremento de la subcontratación de empresas; predominio del capital financiero, y una intervención gubernamental muy limitada en lo económico.

En la década de los setenta emerge con fuerza la ideología "neoliberal", que retoma diferentes argumentos y teorías para demostrar que la intervención del gobierno, en lo económico, es innecesaria y contraproducente. El llamado neoliberalismo creó las condiciones para ampliar los espacios de valorización del gran capital y recuperar su tasa de ganancia; por tanto, ha sido una ideología a favor del capitalismo de los oligopolios. A partir de esta ideología se ha atacado fuertemente a los sindicatos, ya que se considera que las reinvindicaciones por salarios mínimos, protección contra el despido, etc., son distorsiones para el mercado de trabajo que afectan a la inversión privada. Los principales agentes de este proceso de globalización con orientación neoliberal son las empresas transnacionales, y en su lucha por borrar las barreras para la libre movilización de capitales y mercancías han puesto en crisis a los estados-nacionales.

El proceso de globalización de orientación neoliberal incrementa los niveles de dependencia económica de los países subdesarrollados. La actual competencia, a nivel mundial, está basada en el conocimiento, en la capacidad de innovar tecnológicamente, y para ello lo crucial es contar con trabajadores altamente calificados. Lo anterior agudiza la desventaja de las empresas nacionales para competir internacionalmente en países como los centroamericanos: La mayoría de las empresas de la región se caracterizan por un considerable retraso tecnológico, a lo cual contribuyó el modelo de industrialización por sustitución de importaciones y la situación de inestabilidad política de los años ochenta.

Además, una parte importante de la fuerza de trabajo en la región tiene niveles bajísimos de escolaridad y no cuenta con las habilidades y conocimientos adecuados para ajustarse a la nueva tecnología. Más aún, la actual revolución tecnológica requiere de menos horas de trabajo vivo y de trabajo altamente calificado, lo que choca con la sobreabundante oferta de trabajo no calificada que existe en la región. De ahí que la forma en la cual nuestras economías se inserten en las cadenas internacionales de producción queda supeditada, en gran parte, a las conveniencias e intereses de las grandes empresas multinacionales.

Este nuevo marco económico representa un enorme reto a los trabajadores y, en especial, a los sindicatos. El desafío es pasar de una posición meramente defensiva de derechos conquistados en el pasado a ser un actor activo en la negociación de los cambios en los métodos de organización del trabajo, que deben operarse en las empresas de la región. Los sindicatos deben ampliar sus agendas de negociación más allá del salario y las prestaciones laborales, incluyendo aspectos tales como capacitación, calidad de la produccción, control de ritmos de trabajo, incrementos de productividad, tamaño de la jornada de trabajo, apèrtura de nuevos turnos de trabajo, etcétera. El gran objetivo sería alcanzar un pacto con los empresarios que posibilite un incremento en la productividad y mejore tanto las posibilidades de una inserción exitosa en los mercados intemacionales como los ingresos de los trabajadores.

Ante la amenaza de perder derechos laborales, como salarios mínimos, seguridad social, negociaciones colectivas, los sindicatos deben plantear que los derechos laborales constituyen derechos sociales mínimos que garantizan la integración de los más débiles, quienes corren el peligro de quedar excluidos por el funcionamiento de un mercado sin control social. Este punto cobra especial importancia en Centroamérica debido a que el sector "transables nuevos" - las maquilas están dentro de este sector-, que se muestra muy dinámico en la generación de empleo, ha sido cuestionado por no garantizar derechos laborales básicos. Así, los sindicatos podrían levantar la bandera de un pacto social que reclame una "ciudadanía social"12, según la cual los derechos laborales sean parte de los derechos sociales mínimos que el Estado deba garantizar. Sin embargo, la lucha por este pacto no debe ser sólo de los sindicatos de

12. La "ciudadanía social" es un término utilizado por Pérez Sáinz y Allen Cordero (1997, p. 82) y lo consideran como uno de los retos cruciales para los sindicatos. 
una nación, sino de movimientos sindicales internacionales dado el actual contexto de globalización y la pérdida de poder de los estados-nacionales.

En relación con la breve caracterización de los mercados laborales en Centroamérica, cabe destacar los siguientes aspectos de cara a señalar algunos retos para las organizaciones de trabajadores: (a) la mayoría de la población económicamente activa sigue empleada en los sectores de subsistencia agrícola e informal; (b) la tendencia a una creciente feminización de la PEA; (c) el dinamismo del sector "transables nuevos", que incluye a la maquila, en la generación de empleo; (d) la mayoría de la PEA ha sufrido un deterioro de sus ingresos reales en los primeros años de la implementación de los programas de ajuste estructural.

Las organizaciones de trabajadores deben diseñar estrategias para poder integrar a los trabajadores en los sectores de subsistencia agrícola e informal. Esto supone ciertas dificultades, entre otras razones porque estos trabajadores se encuentran muy atomizados y además no se trata de la misma situación de los asalariados formales. Un importante punto común de reinvindicación se encuentra en la lucha por una "ciudadanía social", que posibilite integrar a los sectores más débiles y excluidos del mercado.

La tendencia a la creciente feminización de la Población Económicamente Activa presenta el reto de incorporar una agenda especial para tratar discriminaciones contra las mujeres, como lo son la diferencia salarial en relación con los hombres, mayor inseguridad en el empleo, acoso sexual, carencia de prestaciones como guarderías, etc. Por otro lado, en las maquilas se observa la continuidad del "taylorismo", una participación muy importante de las mujeres, la importancia de su localización territorial y, en algunos casos, irrespeto a los derechos laborales básicos ${ }^{13}$. Pero ante las prácticas tayloristas, las organizaciones laborales deben introducir temas, como el control del ritmo del trabajo, la productividad del trabajo y los salarios, métodos al-

ternativos de organización de la producción, por ejemplo, calidad total.

Dada la importancia de la localización territorial de las maquilas en zonas francas, los sindicatos deben incorporar nuevas formas de organización de carácter territorial e incorporar en sus agendas el tema del desarrollo local ${ }^{14}$. La importancia de la Población Económicamente Activa femenina refuerza la necesidad de elaborar una agenda específica para tratar los problemas laborales más importantes de las trabajadoras.

No podemos omitir que las organizaciones sindicales en la región se encuentran muy debilitadas y con serios problemas de organización intema. No es un objetivo, en este artículo, profundizar en las causas de la crisis del sindicalismo, pero deseamos resaltar que de cara al fortalecimiento de la democracia y el desarrollo socioeconómico de la región, se requiere del fortalecimiento de las organizaciones de los trabajadores para que puedan asumir los retos ya señalados.

Finalmente, los gobienos de la región tienen una enorme deuda socioeconómica con los trabajadores. Una prioridad de las agendas gubernamentales debería ser contribuir a la generación de empleo y mejorar los ingresos reales de la mayoría de la población económicamente activa. Desde nuestro punto de vista, los gobiernos deben tener un rol muy activo por medio de programas de generación 
de empleo y capacitación —que han sido muy limitados en la región-, orientados a ofrecer las habilidades y los conocimientos para que los trabajadores puedan operar las nuevas tecnologías y así se incrementen sus niveles de productividad. Para que esto sea posible se requiere de un creciente movimiento mundial que proponga un nuevo paradigma de desarrollo, centrado en el ser humano, que se contraponga a la ideología "neoliberal" y redefina el rol del Estado, que debe garantizar la vigencia de unos derechos sociales mínimos que incluya a los derechos laborales. Las organizaciones de los trabajadores son uno de los principales agentes que deben luchar por un desarrollo incluyente.

\section{Bibliografía}

Anner, M. “'Hacia la sindicalización de los sindicatos?". ECA, 573-574. San Salvador: Universidad Centroamericana "José Siméon Cañas" (UCA), 1996.
Arriola, J. y Candray, J. "Derechos prohibidos: negociación colectiva y sindicatos en El Salvador". Serie documentos de investigación, No. 1. San Salvador: Universidad Centroamericana "José Siméon Cañas" (UCA), Departamento de Economía, 1994.

Elson, D. et. al. Crecer con la müjer: oportunidades para el desarrollo económico centroamericano. San José: Embajada Real de los Países Bajos, 1997.

Fundación Nacional para el Desarrollo (FUNDE). "La actividad de la maquila en la economía salvadoreña". Actualidad económica, 3, enero, 1996.

Fundación Obrero Empresarial Salvadoreña (FOES). Los trabajadores de frente al futuro y al desarrollo: retos y propuestas para el desarrollo desde la perspectiva del sector laboral salvadoreño. San Salvador, 1997.

Funkhouser, E. y Pérez Sáinz, J. "Ajuste estructural, mercado laboral y pobreza en Centroamérica: una perspectiva regional". En Mercado laboral y pobreza en Centroamérica: ganadores y perdedores del

Nota del autor: el texto sobre los "Sectores Laborales" que se presenta a continuación ha sido tomado de E. Funkhouser, y J. Pérez Sáinz (1998, pp. 339-343). En estos párrafos, los autores definen los sectores laborales que han utilizado para analizar los principales cambios en los mercados laborales de los cinco países de Centroamérica, durante los primeros años del ajuste estructural. Ellos han partido de la propuesta de segmentación del PREALC y han utilizado las variables de categoría de ocupación, ocupación principal, rama de actividad y tamaño de empresa para definir los sectores laborales.

Sector Agrícola Moderno: comprende a patronos y asalariados en la agricultura y minería, que trabajan en empresas de cinco o más personas. También incluyen los profesionales de estas dos ramas de la actividad económica, sin importar el tamaño de la empresa.

Sector de Subsistencia Agrícola: comprende a todos aquellos que trabajan en la agricultura y minería en empresas de cuatro o menos personas, excluyendo a los profesionales y técnicos relacionados con el sector agrícola moderno.

Sector Formal: comprende a los patronos y asalariados de todas las ramas de la actividad económica - con la excepción de agricultura y minería- en empresas de diez o más personas.

Sector Informal Urbano: comprende a aquellos que trabajan en todas las ramas de la actividad económica —con excepción de la agricultura y la minería- en empresas de cuatro o menos personas. También se excluye a los profesionales y técnicos que laboran en el sector formal.

Sector Público: comprende a todos los trabajadores públicos, abarcando todos los tamaños de las empresas.

Sector Empleo Doméstico: sólo se ha utilizado en los casos de El Salvador y Honduras, porque existen como categoría ocupacional diferenciada en sus estadísticas laborales.

Sector Transables Nuevos: los autores lo utilizaron sólo en tres países, Costa Rica, Guatemala y Honduras, que son los que mostraron un mayor desarrollo en estas actividades durante el período estudiado. Las actividades económicas comprendidas son: exportaciones agrícolas no tradicionales, industria de maquila (y zonas francas) y turismo. En el caso de la maquila se tomaron las empresas con 10 y más personas, mientras que para la agroexportación y el turismo se tomaron todos los tamaños de empresas.

Sector de Inclasificables: comprende a las personas ocupadas que no se encuentran asignadas en los sectores antes definidos.

Sector de Desempleados: comprende a los cesantes y a quienes buscan por vez primera empleo. 
ajuste estructural. San José: FLACSO-SSRC, 1998.

González, A. "Orden mundial y liberación”. ECA, 549. San Salvador: Universidad Centroamericana "José Siméon Cañas" (UCA), 1994.

Hirsch, J. "Fordismo y posfordismo: la crisis social actual y sus consecuencias". En Bonefeld, W. y John Holloway (comp.): ¿Un nuevo Estado?: debate sobre la reestructuración del Estado y el capital. México: Editorial Cambio XXI, 1994.

Ibistate, J. “¿Es ético canonizar la globalización?”. $E C A, 583$. San Salvador: Universidad Centroamericana "José Siméon Cañas" (UCA), 1997.

Jiménez, A. "La globalización y las nuevas relaciones de interdependencia compleja en el sistema económico internacional". Realidad, revista de Ciencias Sociales y Humanidades, 62. San Salvador: UCA, 1998.

Lazo, J.F. "El impacto de la nueva revolución tecnológica en el tercer mundo". Realidad, revista de Ciencias Sociales y Humanidades, 62. San Salvador: UCA, 1998.

Nowalski, J.; Morales, P. y Berliavsky, G. Impacto de la maquila en la economía costarricense. Mimeo, San José: Fundación Ebert, 1994.

Pérez, N.C. y Varela, C. "Impacto socioeconómico de las maquiladoras y las zonas francas en Honduras". Informe (San José, OIT), 1995.
Pérez Sáinz, J. y Cordero, A. Globalización, empleo y políticas laborales en América Central. San Salvador: Proyecto Escuela de Formación Sindical en Centroamérica, auspiciado por la Fundación Paz y Solidaridad, Centro de Estudios del Trabajo (CENTRA) y la Cooperación Española, 1997.

Pleitez, R. "La doctrina del neoliberalismo y el proceso de globalización en los países subdesarrollados". Ponencia presesentada en el Colegio de Profesionales de Ciencias Económicas de El Salvador, próximo a publicarse en Realidad, revista de Ciencias Sociales y Humanidades de la UCA, 1998.

Pleitez, R. "Los problemas de concertación económicosocial que presentan los programas de ajuste estructural". Realidad económico-social, 19-20. San Salvador: UCA, 1991.

Sebastián, L. "El neo-liberalismo: una negación del liberalismo". Realidad económico-social, 11. San Salvador: UCA, 1989.

Sebastián, L. "La revolución antiliberal". El País, 13 de mayo de 1998.

Tenorio, M. "Mundialización y Estados-naciones: un acercamiento conceptual". Realidad, revista de Ciencias Sociales y Humanidades, 53. San Salvador: UCA, 1996.

Vidal Villa, J. Mundialización: diez tesis y otros artículos. Barcelona: Icaria editorial S.A., 1996. 